$\xi=$ 离

\title{
Neuro-radiology: a new gold standard investigation for management of central nervous system tuberculosis
}

\author{
Vinod K.S.Gautam * \\ Assistant Professor, Department of Neurosurgery, Institute of Human Behaviour and Allied Sciences, GNCT Delhi, Dilshad Garden, \\ Delhi- 110095, India \\ *Corresponding author E-mail:drvksg@gmail.com
}

\begin{abstract}
Treatment of CNS TB is challenging due to lack of specific biochemical tests and inability to get the pathological sample from deeply located eloquent areas of CNS without causing any neurological deficit. Moreover, it is unnecessary to operate for biopsy in a patient who has presented with a very small granulomatious lesion in brain or spinal cord. In such as situations neuro-radiology helps in managing CNS TB and it may be the only source of establishing diagnosis and evaluating treatment response.

Role of radiological investigation has expanded from the initial diagnosis to the therapeutic interventions. In some Muli-drug- resistant (MDR) CNS TB cases, Stereotaxy or USG or CT guided biopsy helps in obtaining pathological sample and drug sensitivity testing. A regular clinical and neuro-radiological follow-up is mandatory during the entire course of anti tuberculous therapy to take prompt decisions to change ATT and to reduce morbidity and mortality associated with CNS TB.
\end{abstract}

Keywords: CNS Tuberculosis; Neuro-radiology

\section{Introduction}

Timely diagnosis is the key in management of tuberculosis. Traditionally culture and sensitivity testing of the pathological specimen for AFB (Acid-fast fast bacillus), Mycobacterium tuberculosis, has been considered gold standard for diagnosis of tuberculosis. In view of the lack of the sensitivity and specificity of the molecular and biochemical tests and inability to get the pathological sample in most of the cases of central nervous system tuberculosis (CNS TB), there is increased reliance on the radiological investigations. So, this is impossible to get the pathological sample from the deeply located lesions in the eloquent areas of the brain and inside the spinal cord without inflicting any damage to the central nervous system. Moreover, it is unnecessary to operate a patient who has presented with a very small granulomatious lesion in brain or spinal cord. Noninvasive and prompt neuroradiology offers great help in managing CNS TB.

In majority of pulmonary TB cases, a chest X-Ray show the lesion, and it is often easy to get the pathological sputum sample where AFB staining and culture and sensitivity testing is possible. Contrary to this, in most of the CNS TB patients, neuro-radiology is the only source of establishing diagnosis and evaluating treatment response.

\section{Findings of roentgenogram in CNS TB}

With invention of CT scan and MRI, the early diagnosis of CNS TB was possible. But, the $\mathrm{x}$-ray is still of great value in diagnosis, treatment and follow-up of patients with pulmonary or extrapulmonary $\mathrm{TB}$.

\subsection{Chest x-ray}

$\mathrm{X}$-Ray Chest- postero-anterior view (PA view) should be done in all patients of CNS TB. Although X-Ray chest may not be positive for pulmonary TB, however, in some cases it may reveal evidence of tubercular etiology. Various studies have shown that about 12 to 60 percent patients with CNS TB show features suggestive of concomitant pulmonary TB on their chest x-rays [Davis et al. 1993, Tariq \& Sheikh 1994, Alsoub 1998, Girgis et al. 1998, Venugopal et al. 2008, Gautam et al. 2013].

Although, a chest $\mathrm{x}$ ray is mandatory in all patients suspected to have $\mathrm{TB}$, roentgenogram of different other areas may also become indispensable in certain cases of extrapulmonary TB. X- Ray of the skull may reveal a lytic lesion in tubercular osteomyelitis of the skull bone [Gautam et al. 2013, Gautam \& Singh 2014]. Sometimes CT scan of the chest or abdomen is required.

\subsection{Skull $x-$ ray}

If patient presents with a cold abscess of the scalp or a bony lesion or defect in the skull, X- Ray skull both antero-posterior and lateral view ( Fig 1 A \& B) should be advised, which may reveal a lytic lesion with irregular margins suggestive of tubercular osteomyelitis. If there is an associated cold abscess, an anti-gravity aspiration of the pus can be done and pus should be sent for AFB stain, Gram stain, PCR for TB, AFB culture and sensitivity [Gautam et al. 2013]. Skull X-ray is not done unless osteomyelitis of skull bone is suspected. 


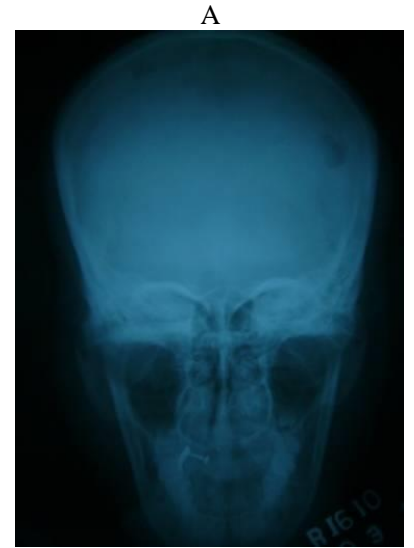

B

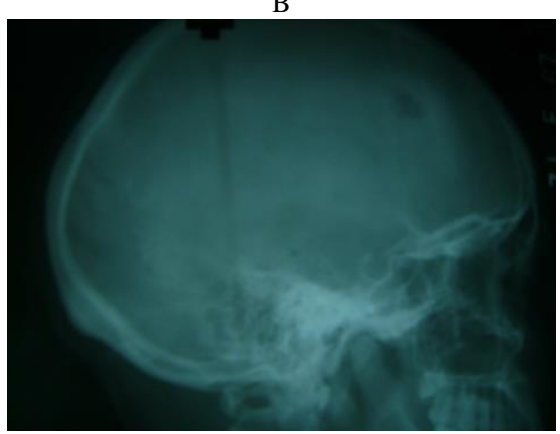

Fig. 1: Anteroposterior ( A) and Lateral View ( B ) of Skull Showing A Defect in the Craniaum

\subsection{X-ray of spine}

Plain radiography is insensitive for the early detection of vertebral TB. Disk space narrowing may be quite subtle and vertebral involvement is not detected until at least $50 \%$ of the trabecular bone is lost. Plain radiography of tuberculous spondylodiscitis may demonstrate erosion or destruction of pedicle, loss of vertebral height or collapse of vertebral body, disc space narrowing ( Fig.2), erosions, indistinction of the end plates, paravertebral masses, and soft-tissue calcifications, kyphosis or scoliosis of spine [Moore \& Rafii 2001, De Backer et al. 2005, Gautam \& Singh 2014].

Even with the advent of MRI, the x-ray of the spine (AP \& Lateral view) is still relevant and should be done in all patients suspected to have tubercular spine.

Some specific X-rays may be done as and when required. For example, in tuberculosis of the cranio-vertebral (CV) junction, plain X-rays should be obtained for all patients, including X-ray $\mathrm{CV}$ junction -lateral view (neutral, flexion, extension), and open mouth view.

Plain x-rays may reveal enlargement of the retropharyngeal space at $\mathrm{C} 1$ and $\mathrm{C} 2$, atlantoaxial dislocation (AAD) and other bony abnormalities.

Flexion, extension and neutral views of spine are helpful to diagnose the mobility of the affected spine, instability of the spine and need for spinal fixation, irreducible-type AAD if atlantodental space is not reduced after putting the patient on traction in extension, and spondylolisthesis.

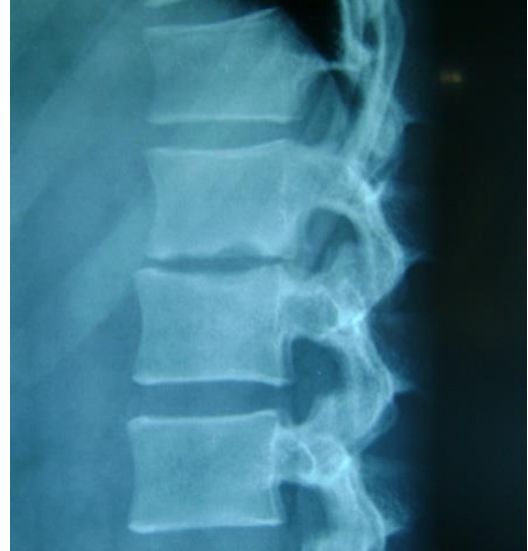

Fig. 2: Lateral View of the Thoraco-Lumbar Spine Showing Diminution of Intervertebral Disc Space.

\section{Newer diagnostic tools}

Since invention of Roentegogram, many advances have occurred in neuroradiology. Myelogram, angiography, bone scan, CT scan, MRI, Positron Emission Tomography (PET) CT, PET MRI, SPECT and many more. But, all these investigative modalities may not be relevant in all cases. Judicous decision to use a particular investigaton should be based on the clinical presentation of each patient. Sometimes, CNS tuberculoma may mimic brain metastasis. Similary, cervical lymphadenopathy may be due to cancer. In such cases, PET CT may be valuable [Gautam et al. 2014]. Bone scan should not be advised as a routine investigation for the diagnosis of CNS TB [ Gautam et al. 2014]. Computerized tomography $(\mathrm{CT})$ and magnetic resonance imaging (MRI) have vastly improved the diagnostic accuracy of tuberculosis of central nervous system.

NCCT scan of brain may be normal in the initial phase of TBM. Commonly identified neuroradiological features of TBM on contrast enhanced CT scan of the brain reveals include abnormal meningeal enhancement, leptomeningeal enhancement at sylvian fissure, tentorium, obliteration of basal cisterns, basal meningeal enhancement ( basal exudates), granulomas in the basal meninges and ependymitis, hydrocephalus, calcifications, ring enhancing granulomas, abscess and infarctions in the supratentorial brain parenchyma, cerebellum and brain stem (Fig.3\&4) [Gupta et al. 1994, Gupta et al. 1999, Gupta 2002, Trivedi et al. 2009].

Contrast-enhanced MRI is generally considered to be superior to $\mathrm{CT}$ in detecting and assessing CNS tuberculosis [Offenbacher et al. 1991, Kioumehr 1994, Jinkins 1995, Bernaerts et al. 2003]. MRI with contrast detects the extent of pachymeningitis, leptomeningeal disease and parenchymal abnormalities.

In an attempt to establish CT criteria for the diagnosis of CNS tuberculosis, Kumar et al. identified basal meningeal enhancement, ventriculomegaly, tuberculoma, and infarcts as characteristics to distinguish CNS tuberculosis from pyogenic meningitis and proposed that basal meningeal enhancement, tuberculoma, or both were $89 \%$ sensitive and $100 \%$ specific for TBM [Kumar et al. 1996].

A

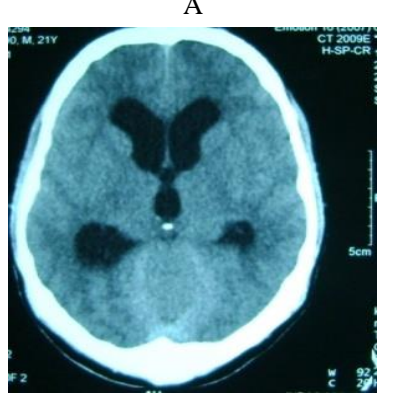

Fig. 3: CT Scan of Brain Axial Views: A. Noncontrast and B. with Contrast, Showing Meningeal Enhancement and Ventriculomegaly.

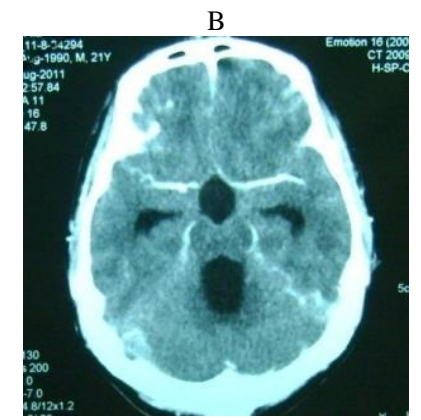


The early changes in ventriculomegaly is suggested by the blunting of the frontal horns of the lateral ventricle. The temoral horns become visible and size becomes more than 2 to $3 \mathrm{~mm}$. Third ventricle no longer remains slit like and becomes globular. The sulci become effaced in the obstructive hydrocephalus (Fig.3). Hypodensity in the brainstem on NCCT scan head could be due to infarct or edema in the brain stem (Fig. 4, 6) [Greenberg 2010]. Przybojewski et al. evaluated nine of these distinct CT criteria for basal meningeal enhancement and suggested that the presence of four criteria were highly specific and that having more than one criteria was 91\% sensitive for TBM [Andronikou \& Wieselthaler 2004, Przybojewski et al. 2006].

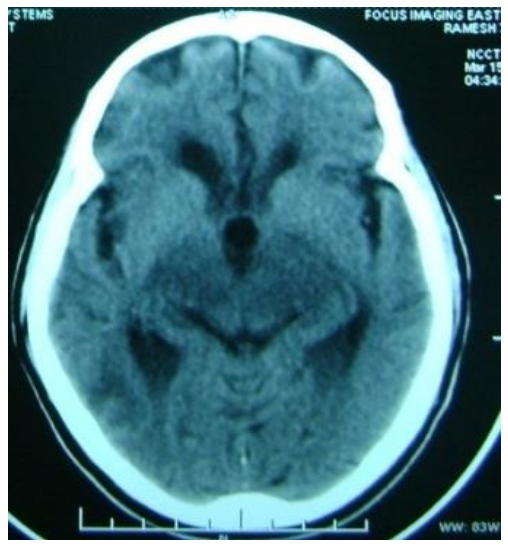

Fig. 4: NCCT Scan of Head Showing Hypodensity in the Brain Stem.

Magnetization transfer ( MT) imaging is considered to be superior to conventional spin echo (SE) sequences for imaging abnormal meninges, which appear hyperintense on pre contrast T1W MT images and show further enhancement on post contrast T1W images. In addition, MT ratio quantification helps in predicting the etiology of meningitis [Gupta et al. 1994, Gupta et al. 1999, Gupta 2002, Kamra et al. 2004, Trivedi et al. 2009]. Visibility of the inflamed meninges on precontrast T1W MT images with low MTR is specific for TBM, helping in differentiating it from other nontuberculous chronic meningeal infections [Kamra et al. 2004]. MR spectroscopy of the tubercular lesion shows lipid peak in cases of tubercular granulomatous lesion (Fig.5). The combination of MRS with MT MRI may be of value in the diagnosis of TBM [Trivedi et al. 2009].

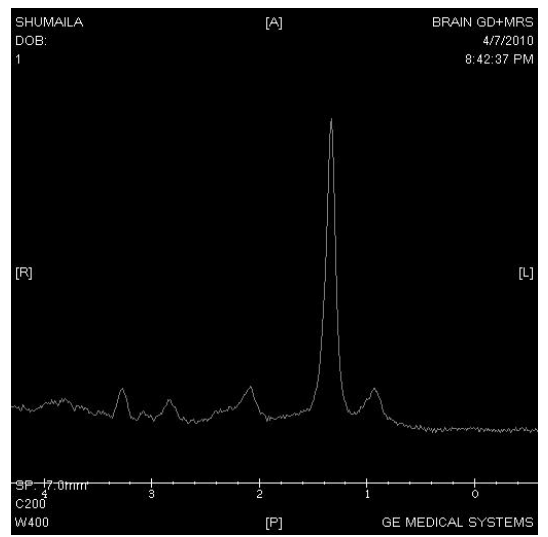

Fig. 5: MR Spectroscopy of Brain Lesion Showing Lipid Peak.

\subsection{Neuroradiological findings in hydrocephalus}

Hydrocephalus encountered in TBM can be broadly divided into two types: (1) communicating type, which is common, secondary to an obstruction of the basal cisterns by inflammatory exudates and (2) obstructive type, which is less common and either secondary to a focal parenchymal lesion causing mass effect or due to the entrapment of a part of the ventricle by granulomatous ependymitis [Trivedi et al. 2009, Tandon et al. 1988]. Periventricular hyperintensity on proton density and $\mathrm{T} 2 \mathrm{~W}$ images is due to the seepage of the CSF fluid across the white matter and usually suggests hydrocephalus under pressure, which is an indication for CSF diversion surgery to decompress the ventricular system [Trivedi et al. 2009, Singh et al. 2008].

The common site of ventricular obstruction in obstructive hydrocephalus is aqueduct causing enlargement of the lateral and third ventricle. However, there may fourth ventricular outlet obstructon (foramen of Luschka and formen of Magendie) leading to panventriculomegaly. Sometimes, there may be enlargement of only one ventricle (Fig. 8-12).

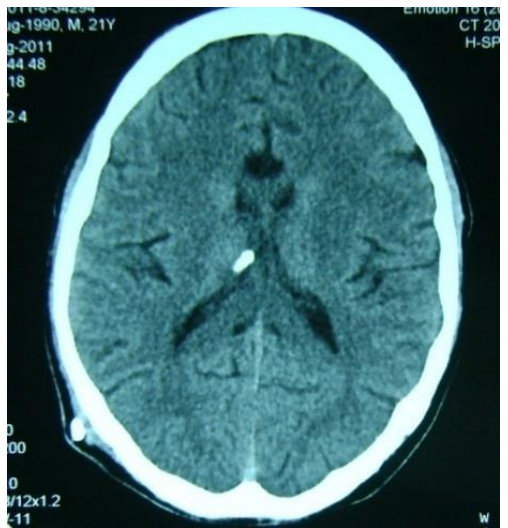

Fig. 6: CT Scan Brain Showing Shunt in Situ and Diminished Ventricle Size.

On CT scan, hydrocephalus can be assessed with Evans ratio and periventricular lucency. Periventricular lucency, a term sometimes used to describe the hypodensity around the lateral ventricle in patients of hydrocephalus is basically a misnomer, as the term lucency is used in $\mathrm{X}$ ray report. The term should be periventricular hypodensity, which indicates trans-ependymal flow of CSF. This is a sign of raised intraventricular hydrostatic pressure and raised intracranial pressure and indicates need for prescribing cerebral decongestants, including acetazolamide or necessitates some kind of CSF diversion procedure. In cases of TBM, periventricular hypodensity could also be due to spread of an inflammatory process.

The easy way to recognize ventriculomegaly on the CT is that there will be enlargement of the ventricular size, the frontal horns may look blunted; the third ventricle may become globular; the cisterns may get obliterated in case of obstructive hydrocephalus; the temporal horns of the lateral ventricle may look prominent and more than 2 to $3 \mathrm{~mm}$ in size [Greenberg 2010]

In newborn, infant, and in children below age of 18 months, in whom the anterior fontanel is still open, cranial ultrasound is safe investigation to assess the progression of hydrocephalus.

\subsection{Neuroradiological findings in vasculitis}

The adventitial layer of small and medium-sized vessels develops changes similar to those of the adjacent tuberculous exudates. The intima of the vessels may eventually be affected or eroded by fibrinoid-hyaline degeneration. In later stages, the lumen of the vessel may get completely occluded by reactive subendothelial cellular proliferation. Ischemic cerebral infarction resulting from the vascular occlusion is a common sequelae of tuberculous arteritis. The middle cerebral and lenticulostriate arteries are most commonly affected [Dastur et al. 1970, Trivedi et al. 2009]. Diffusion-weighted MR imaging helps in the early detection of this complication [Shukla et al. 2008, Trivedi et al. 2009].

\subsection{Neuroradiological Findings in pachymeningitis}

An unusual presentation of CNS TB is isolated involvement of the dura, known as pachymeningitis, which is distinct from the inflammation of the dura adjacent to an intraparenchymal tuberculoma [Kumar et al 1996, Brismar 1996, Goyal et al 1997, Trivedi et al 2009]. It consists of either isolated dural involvement or pial 
or parenchymal involvement that is secondary to a dura-based lesion. As in the case of TBM, tuberculous pachymeningitis may also result from hematogenous spread of the bacilli. Pachymeningitis may exist as focal or diffuse involvement of the dura [Trivedi et al. 2009, Offenbacher et al .1991, Brismar et al. 1996, Goyal et al. 1997]. Pachymeningitis will appear as thickened dura, which will be hyperintense on T1W1 contrast image on MRI (Fig.7).

\subsection{Neuroradiological findings in brain tuberculoma}

Brain Tuberculoma, a space-occupying mass of granulomatous tissue [Tandon \& Pathak 1973, Trivedi et al. 2009] forms a large percentage of intracranial mass lesions in the developing countries. Tuberculomas may be single or multiple, and can be seen anywhere in the brain parenchyma [Gupta \& Lufkin 2001, Trivedi et al. 2009].

On CT scan, tuberculoma may also appear as hypodense lesion without of proportion edema in cerebritis stage. Mature tuberculoma shows either ring or nodular enhancement with perilesional edema. Caseating tubercular granloma on CECT shows a rimenhancing lesion with a caseating hypodense center.

A variable degree of vasogenic oedema surrounds the lesions, which is better appreciated on T2-weighted MR images. Enhancement of the granulomas on the post-gadolinium scans improves their conspicuity and enables their differentiation from the adjacent vasogenic edema [Khoo et al. 2003].

The "target sign" (central nidus of calcification surrounded by a ring of enhancement) was once considered to be pathognomonic for tuberculoma [Bernaerts et al. 2003], but this has recently been called into question [Bargallo et al. 1996]. The radiographic presentation of tuberculoma depends largely on whether the lesion is noncaseating, caseating with a solid center, or caseating with a liquid center; the degree of edema surrounding the tuberculoma is thought to be inversely proportional to the age of the lesion [Bernaerts et al. 2003]. While new or enlarging tuberculoma may occur in some patients despite adequate ATT, the activity of tuberculoma can generally be assessed by the degree of contrast enhancement on follow-up CT or MRI studies [Bernaerts et al. 2003].

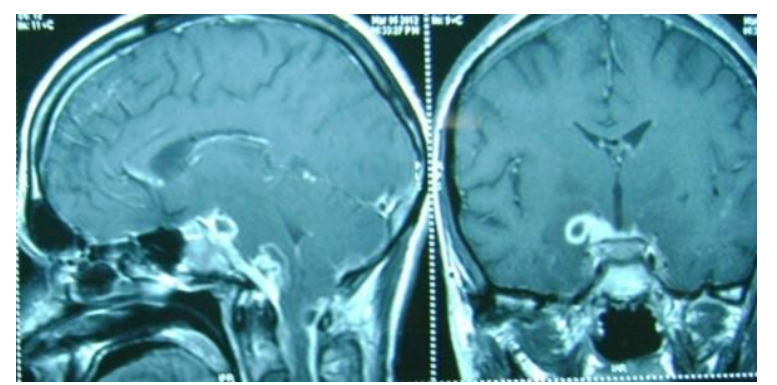

Fig. 7: Mri Images Showing Basal Exudates, Granu-Lomas, Basal Exudates, Features of Vasculitis \& Edema

MRI features of tuberculomas may be similar to those of other intracranial focal lesions, like neurocystycere-cosis, fungal granulomas, glioma, metastasis, chronic pyogenic brain abscess, and lymphomas. So, other in-vestigations are used in conjunction with MRI brain with contrast with MR spectroscopy with quantitative MT imaging to diagnose lesions, which constitute a spectrum of differential diagnosis of tuberculomas [Trivedi et al. 2009, Gupta et al. 1990, Gupta et al. 1993, Poptani et al. 1995, Gupta et al. 1995, Gupta et al. 1996, Gupta \& Roy 1999].

\subsection{Neuroradiological findings in tuberculous brain abscess}

Tuberculous brain abscess is a relatively rare condition constituting 4-7\% of the total number of CNS TB cases in developing countries. According to the criteria of Whitener, tuberculous abscesses should show macro-scopic evidence of abscess formation within the brain parenchyma and should offer histological confirmation that the abscess wall is composed of vascular granulation tissue containing both acute and chronic inflammatory cells and Mycobacterium. [Whitener 1978, Trivedi et al. 2009].

Tuberculous abscesses appear as large, solitary, and frequently multiloculated, ring-enhancing lesions with surrounding edema and mass effect on MRI. [Farrar et al. 1997, Trivedi et al. 2009] MTR quantification from the rim of the abscess has helped in the differential diagnosis of tuberculous from pyogenic abscesses.[ Gupta et al. 2001, Trivedi et al. 2009]. High lipid-containing M. tuberculosis bacilli are probably respon-sible for the significantly lower MTR values in the rim of tuberculous abscesses compared with pyogenic ab-scesses. DWI in tuberculous abscesses shows restricted diffusion with low apparent diffusion coefficient (ADC) values, probably a result of the presence of intact inflammatory cells in the pus [Trivedi et al. 200913, Gautam et al. 2013, 2014].
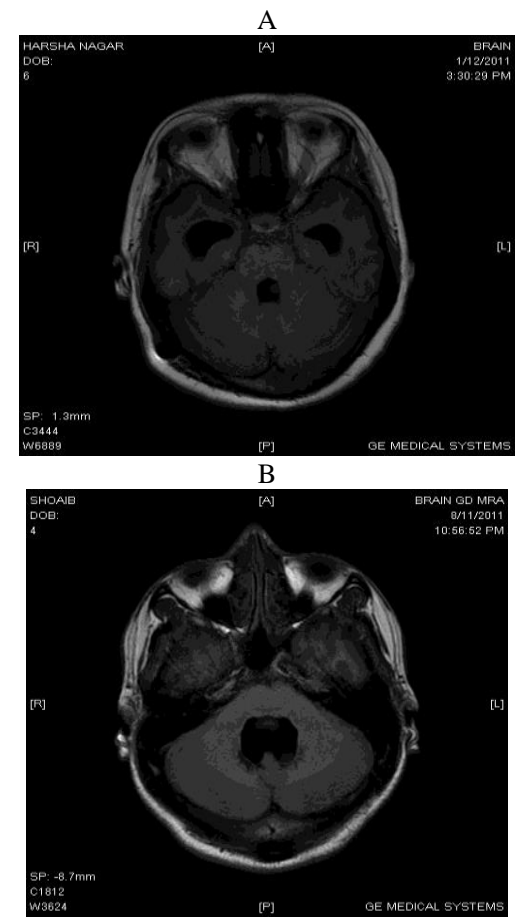

Fig. 8: A. MRI Brain T1W1 Axial View Showing Enlarged Temporal Horns of the Lateral Ventricle, B. $4^{\text {th }}$ Ventricular Enlargement
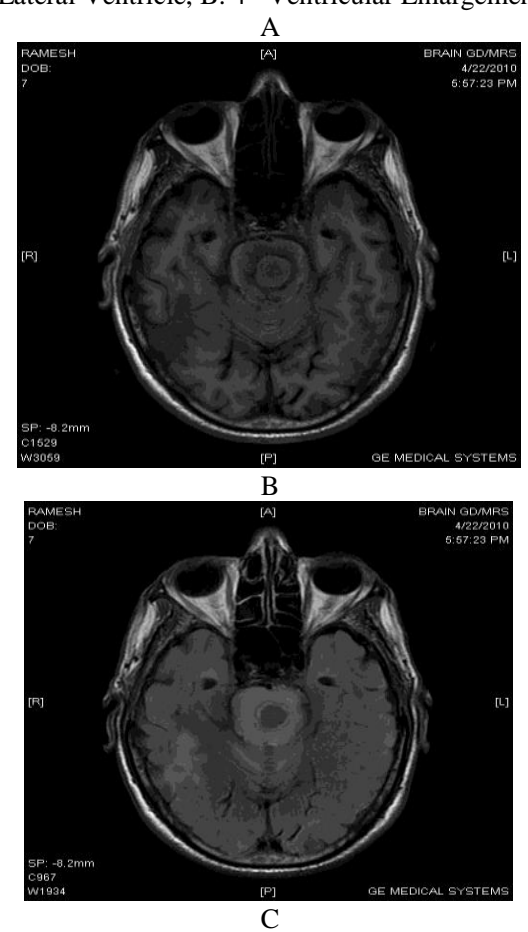


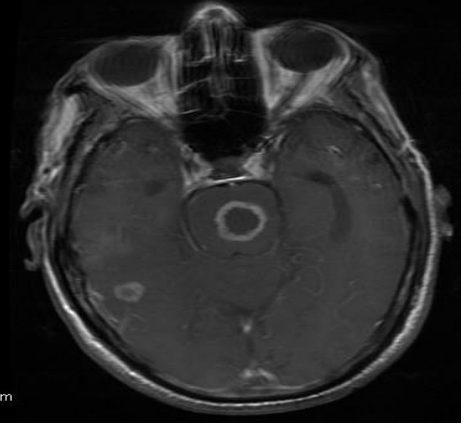

Fig. 9: A. MRI Brain T1W1 Axial View Showing A Mixed Intensity Rounded Lesion in Pons

b. MRI brain T2W2 image showing brain stem lesion showing caseous necrotic centre appearing hypointense on T2 associated edema

c. MRI Brain with T1WContrast (axial view) showing Brain stem Tuberculoma nonenhancing hypointense center and with homogenous intense contrast enhancement of the peripheral rim and perilesional edema.

A

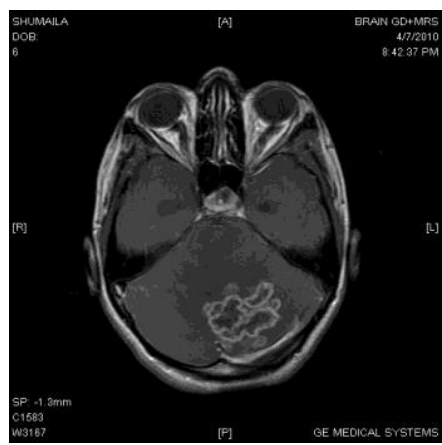

B

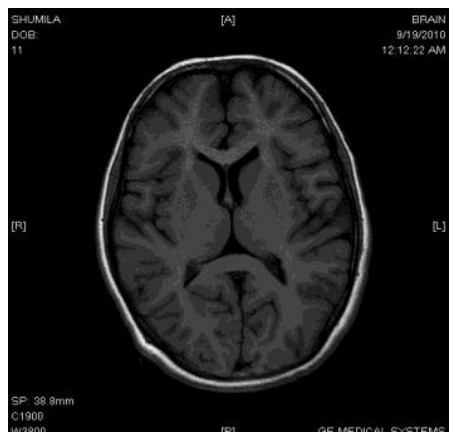

Fig. 10: A.MRI Brain T1W1 Axial View with Contrast Showing Posterior Fossa Conglomerate Tuberculoma and Enlargement of Temporal Horns of Lateral Ventricle, B. Post-Operative Follow Up MRI Brain T1W1 Axial View Showing Disappearance of the Tuberculomas, Absence of the Hydrocephalus and No Shunt in Situ

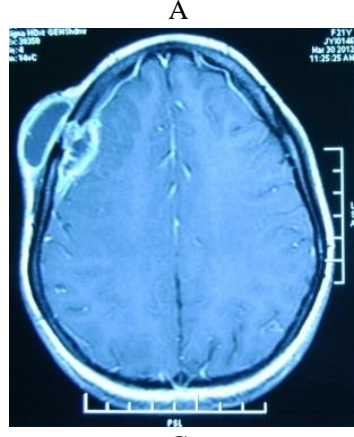

C

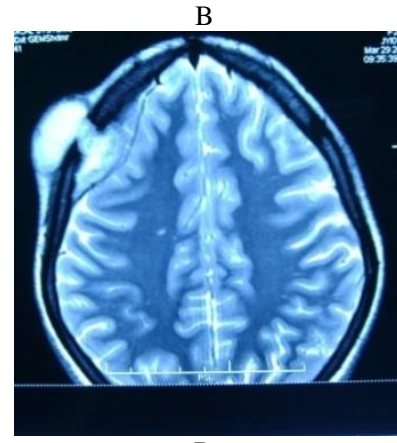

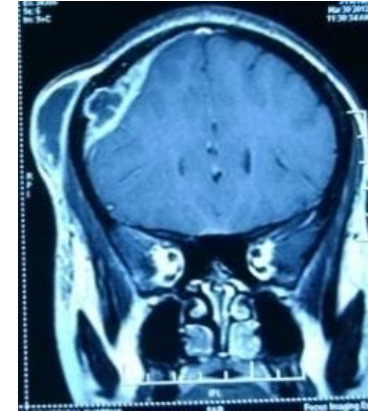

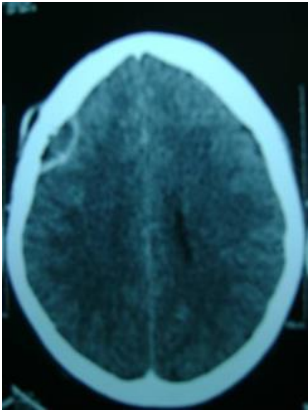

Fig. 11: Calavrial Tuberculosis:

a) T1W Contrast MRI Axial View Showing Hypointense Lesion with Peripheral Contrast Enhancement

b) T2W Axial View Shows Mixed Hyperintense Lesion

c) T1w Contrast Coronal View

d) Contrast Enhanced CT Axial View
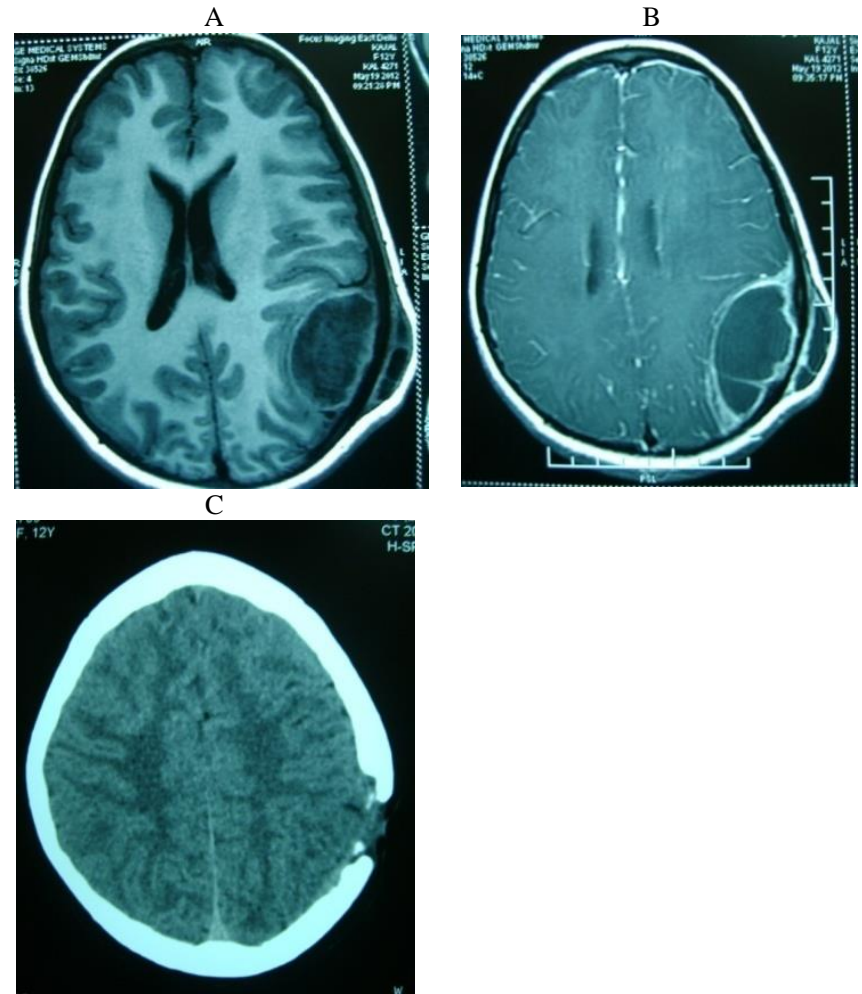

Fig. 12: MRI T1W1 Axial Image of Brain Showing Subgaleal and Extradural Peripherally Enhancing Hypointense Lesion, B. Contrast MRI T1W1 Axial Image of Brain Showing Subgaleal and Extradural Peripherally Enhancing Hypointense Lesion, C. Post Operative CT Scan of the Patient Showing Complete Resolution of the Lesion after Surgery And A Course of ATT.

\section{Role of CT guided stereotaxy}

CT- guided sterotactic surgery helps in retrieving biopsy material from the deep located lesions in the eloquent area of the brain. It may be used either for the diagnostic or therapeutic purposes. It helps in histopathological confirmation of the lesion which remains the gold standard to increase the diagnostic accuracy and to avoid inappropriate treatment . Stereotaxy may also be used for the aspiration of deep seated tuberculous lesion in brain or spine not responding to conservative treatment and decision is warranted to start second line ATT after obtaining drug sensitivity testing of the pathological sample [Ersahin et al. 2010].

\section{Role of ultrasonography}

Ultrasonography (USG) is a noninvasive aid in evaluation of tuberculosus lymphadenopathy. Tuberculous lymphadenitis is the most common form of extra-pulmonary tuberculosis and cervical 
lymphnodes are most frequently involved group among peripheral lymphnodes. [MRC Report 1980, Gupta et al. 2007]. Most of the patients of spinal tuberclosis have concomitant intraabdominal periaortic lymphadenitis and prevertebral pus collection. So, USG abdomen should be a routine dianostic tool for assessing a case of spinal TB.

\section{CT of the spine}

In spinal $\mathrm{TB}$, infection typically commences at the superior or inferior anterior vertebral body corner adjacent to the discovertebral junction, and spreads by subligamentous extension and penetration of the subchondral plate. Later, the lateral and anterior cortices of the vertebral body may become destroyed, leading to cavitation, wedging, collapse, vertebral instability and gibbus or kyphotic deformity. Because the disc is avascular, disc infection is seen late, and results in disc interval narrowing secondary to herniation of the disc into the undermined, collapsed vertebral body. When two contiguous vertebral bodies are involved, the nutrition of the disk is affected [De Backer et al. 2005].

Less often, posterior elements of the spine may be involved. Involvement of the neural arch may occur either alone or in combination with vertebral body lesions (10). Other atypical patterns of tuberculous involvement of the spine consist of infection of a single vertebra or multiple nonadjacent vertebrae ('skip lesions') [Moore \& Rafii 2001].

CT scan of the spine may show the patterns of bony destruction, calcifications in soft tissue or paraspinal abscess and for regions, which are difficult to visualize on plain films, like craniovertebral junction.

CT guided biopsy is another tool to diagnose spinal tuberculosis. CT guided biopsy, thoroacscopic / laproscopic biopsy may add to armmentorum for the definitive diagnosis of tubercular spine [ $\mathrm{Gu}-$ lati \& Gupta 2005]

CT scan of the spine is sometimes required to whether the patient cannot undergo MRI due to claustrophobia, or the presence of pacemaker or ferromagnetic implant in the body.

\section{MRI of the spine}

MRI is the investigation of choice for diagnosis of spinal tuberculosis because of its multiplanar capability, superior tissue contrast, higher sensitivity for detection of early inflammatory bone marrow changes and infiltrative end plate changes in the vertebra. MR imaging is mostly useful in delineating paravertebral, epidural, and intraosseous abscesses and in evaluating the extent of cord compression and the presence of intramedullary lesions (Fig.13\&14) [De Backer et al 2005].

\subsection{Neuroradiological findings in TB myelitis}

The MRI imaging features of TB myelitis are similar to those of cerebritis. Edema of the spinal cord, hyperintensity on T2 weighted image and marginal enhancement on postcontrast $\mathrm{T} 1 \mathrm{~W}$ images [Murphy 1998, Trivedi et al. 2009, Kumar 2005]. The surrounding edema continues to be more extensive than the margins of enhancement. These findings suggest the beginning of intramedullary abscess formation. The central cavitary portions of the intra-axial necrotic areas are seen as hypointense and hyperintense foci on $\mathrm{T} 1 \mathrm{~W}$ and $\mathrm{T} 2 \mathrm{~W}$ images, respectively. Although the abnormalities visible on T2W images subside in several weeks, foci of contrast enhancement on postcontrast images may persist for several months [Murphy 1998, Trivedi et al. 2009].

\subsection{Neuroradiological findings in tuberculous spondyli- tis}

The vertebral bodies are the most commonly involved; the posterior osseous elements, epidural space, paraspinal soft tissue, and intervertebral discs are also involved either secondarily or some- times as the primary area to be first involved [Sharif et al. 1992, Trivedi et al. 2009]. The most commonly involved sites are the dorsal and lumbar spine, especially the thoracolumbar junction. Although the sacrum and cervical spine are the least affected, more than one vertebral level is often involved.
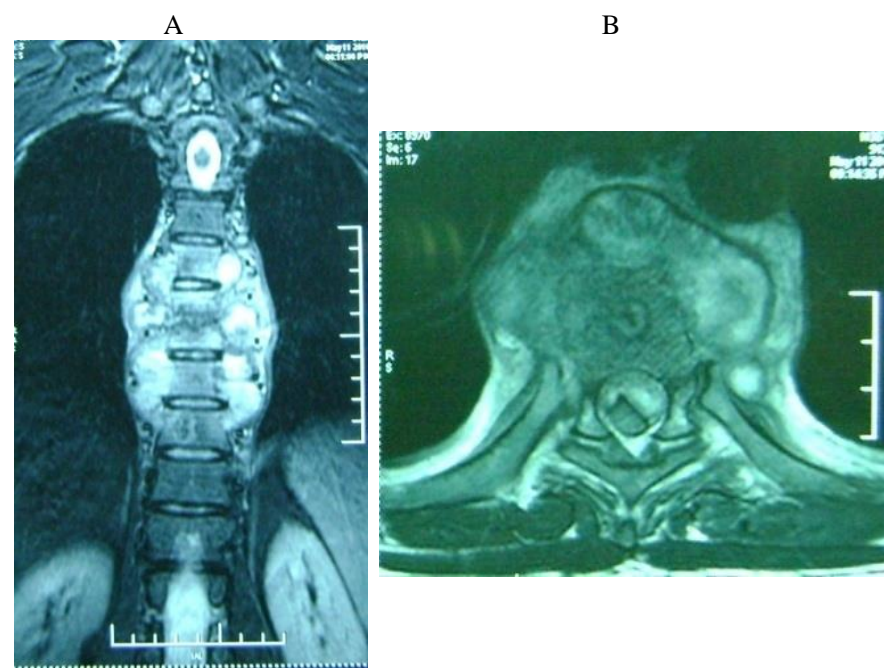

Fig. 13: A. MRI of the Thoracic Spine Coronal View Showing Paravertebral Tubercular Granulation Tissue, B. Axial View Showing Destruction of the Vertebral Body and Compression of the Soinal Cord Due to Epidural Collection of the Tubercular Granulation Tissue

Because of its ability to detect marrow abnormalities before bony destruction, MRI is sensitive for the early detection of tuberculous spondylitis, even in patients with normal radiographs. In the majority of cases, tuberculous spondylitis appears hyperintense on T2W and hypointense on T1W images, showing vertebral body involvement [Sharif et al .1992, Trivedi et al. 2009]. Classic discovertebral involvement can be noted with progression of the disease. Vertebral intra-osseous abscesses, paraspinal abscesses, discitis, skip lesions, and spinal canal encroachment are all readily seen on MR imaging. Reduction in disc height and morphological alteration of the paraspinal soft tissue become apparent during the later stages of infection.

Enhanced MRI studies are useful for characterizing tuberculous spondylitis. Rim enhancement on postcontrast T1W images around intra-osseous and paraspinal soft-tissue abscesses is characteristic of tuberculous spondylitis [Trivedi et al. 2009].

MRI has turned out to be a boon in making a diagnosis, picking up skip lesions, understanding the details of bony and soft tissue involvement, extent of extradural compression, presence or absence of meningeal involvement, cord changes etc.[ Kumar 2005].

Epidural TB lesions generally appear to be isointense to the spinal cord on T1W images and have mixed intensity on T2W images. In postcontrast images, uniform enhancement can be seen. Epidural tuberculous abscess may occur as primary lesions or may be seen in association with arachnoiditis, myelitis, spondylitis, and intramedullary and dural tuberculomas [Murphy 1998, Trivedi et al. 2009].

\subsection{Neuroradiological findings in intradural extrame- dullary (IDEM) tuberculoma}

Intradural extramedullary tuberculoma is rare and signal intensity changes, and meniscus sign may be seen inside the dura and outside the spinal cord. Contrast MRI may delineate the ring enhancing tuberculoma.

\subsection{Neuroradiological findings in spinal arachnoiditis}

The pathophysiology of spinal meningitis is similar to that of TBM; submeningeal tubercle forms during primary infection and ruptures into the subarachnoid space, eliciting mediators of delayed hypersensitivity [Brooks et al. 1954Trivedi et al. 2009]. As 
with intracranial lesions, there is granulomatous inflammation with areas of caseation and tubercles with eventual development of fibrous tissue in chronic or treated cases[Khoo et al. 2003]. Spinal tuberculous meningitis (STBM) may originate in 3 ways; haematogenous spread from a source outside the CNS, caudal extension of intracranial tuberculous basal meningitis and intraspinal extension from osseous or discal TB.

For spinal arachanoiditis, MRI is the modality of choice as delineates leptomeningeal.

disease better.

Tuberculous pus formation occurs between the dura and the leptomeninges and may appear loculated. It appears hyperintense on $\mathrm{T} 2 \mathrm{~W}$ and iso- to hypointense on $\mathrm{T} 1 \mathrm{~W}$ images.

MRI features include CSF loculation and obliteration of the spinal subarachnoid space with a loss of the outline of the spinal cord against CSF in spinal canal on T1 weighted image. Matting of the nerve roots is seen in the lumbar region. Sometimes, unenhanced MRI may appear normal but contrast enhanced imaging will reveal nodular, thick, linear, intradural enhancement, often completely filling the subarachnoid space. So, the key point is that one should suspect spinal arachnoiditis on MRI when T1 contrast enhanced images look like T2 images [aimsnet.org].

In chronic stages of the disease, the postcontrast images may not show any enhancement even when unenhanced images show signs of arachnoiditis.

\subsection{Neuroradiological findings in syringomyelia}

Spinal cord involvement in the form of infarction and syringomyelia may occur as a complication of arachnoiditis. Parenchymal TB myelitis and tuberculoma formation may also occur. Syringomyelia is seen as cord cavitation that typically demonstrates CSF intensity on T1W and T2W images but does not enhance on postcontrast images [Kumar 2005, Trivedi et al. 2009].

Syringomyelia can occur as a complication of arachnoiditis. Syrinx is the lesion inside the spinal cord. It appears hypointense on T1W1 and hyperintense on T2W2 image without contrast enhancement.

\subsection{Neuroradiological findings in intramedullary TB}

Intradural intramedullary involvement is very rare [Jaiswal et al. 2006].

MRI shows low or intermediate signal intensity on T1W images and low signal on T2Wimages (Low signal on T2W images is due to caseous necrosis in the tuberculoma, which has high-protein content). Post Gadolinium study shows ring enhancement. Sometimes associated with changes of myelitis with altered cord signal intensity.
A

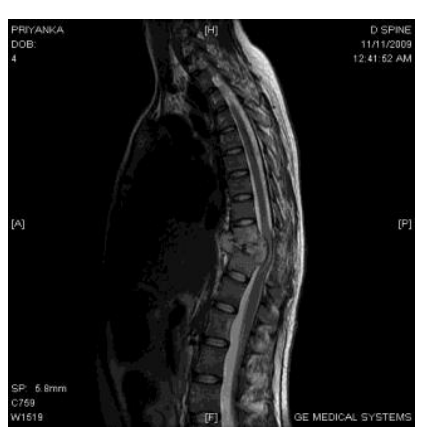

$\mathrm{C}$

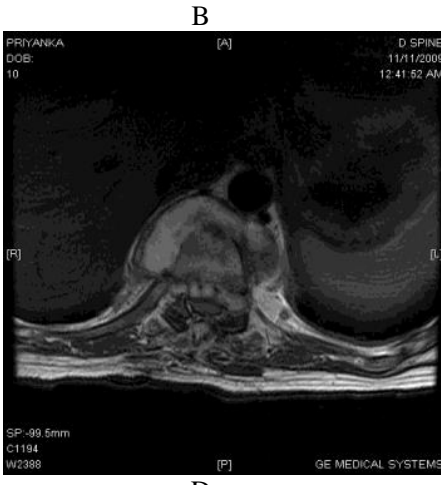

D
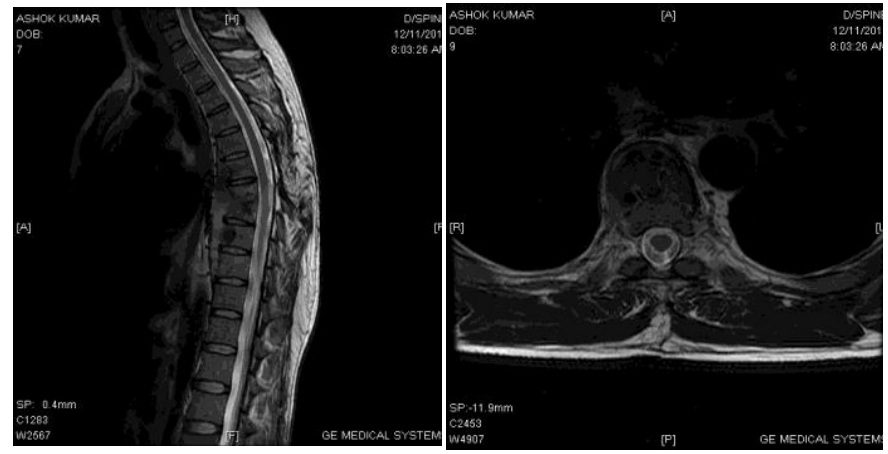

Fig. 14: A \& B: MRI Thoracolumbar Spine Showing Collapse of the Vertebra And Compression of the Spinal Cord Due to Granulation Tissue in the T2W2 Sagittal View, and Axial Views, C \& D: Post-Operative Follow Up MRI Thoracolumbar Spine T2W2 Sagittal and Axial Views Showing Fusion of the Adjoining Vertebrae. Although Granulation Tissue is Still Present, It is Confined Anterior to the Vertebral Body, So there is No Compression Over the Spinal Cord Leading to Complete Neurological Recovery of the Patient.

\section{Conclusion}

Neuroradiological evaluation has become the most important step in the clinical management of CNS TB patients. Role of radiological investigation has expanded from the initial diagnosis to the therapeutic interventions. Stereotaxy, Ultrasound or CT guided biopsy often requires a team approach. With the rise of MDR CNS TB cases, the neuroradiological evaluation and intervention neuroradiology for obtaining pathological sample and drug sensitivity testing is the most important step to start second line ATT for treating MDR CNS TB. This crucial step will minimize the morbidity and mortality of patients affected with tuberculosis of brain and spine. In all cases of CNS TB, a regular clinical and neuroradiological follow-up is mandatory during the entire course of anti tuberculous therapy.

\section{References}

[1] Gautam VKS, Khurana S \& Singh R (2013) Diagnostic and therapeutic challenges in the surgical management of CNS tuberculosis. International Journal of Medicine and Health Sciences, Vol-2; Issue2, 161-169.

[2] Girgis NI, Sultan Y, Farid Z, Mansour MM, Erian MW \& Hanna LS (1998) Tuberculosis meningitis, Abbassia Fever Hospital-Naval Medical Research Unit No. 3.Cairo, Egypt, from 1976 to 1996. American Journal of Tropical Medicine and Hygiene. 58(1), 28-34.

[3] Malik ZI, Ishtiaq O, Shah NH, Anwer F \& Baqai HZ (2002) Analysis and outcome of 30 patients with Tuberculous Meningitis. Pakistan Journal of Medical Research, Vol.41 No.4, 137-141.

[4] Davis LE, Rastogi KR, Lambert LC \& Skipper BJ (1993) tuberculous meningitis in the Southwest United States: a community based study. Neurology; 43(9):1775-8. http://dx.doi.org/10.1212/WNL.43.9.1775.

[5] Tariq M \& Sheikh M (1994) Factors affecting the outcome in tuberculous meningitis. J Surg; 8-9:16-8.

[6] Alsoub H (1998) Tuberculous meningitis:a clinical and laboratory study of 20 patients in Qatar. International Journal of Clinical Practice. 52(5):3004.

[7] Venugopal K, Sreelatha PR, Philip S \& Kumar V (2008) Treatment outcome of neurotuberculosis patients put on DOTS--an observation study from the field. Indian Journal of Tuberculosis. 55, 199-202

[8] Gautam VKS, Khurana S \& Singh R (2013) Primary Calvarial Tuberculosis (PCT) presenting as cold abscess of the scalp: A report of two cases. IOSR Journal of Dental and Medical Sciences (IOSRJDMS), Volume 4, Issue 4, 14-17. http://dx.doi.org/10.9790/08530441417.

[9] Gautam VKS \& Singh R (2013) surgical management of Neurotuberculosis- Review of radiological spectrum. International Journal of Health.1 (1), 1-7. http://dx.doi.org/10.14419/ijh.v1i1.941.

[10] De Backer AI, Mortelé KJ, Vanschoubroeck IJ, Deeren D, Vanhoenacker FM, De Keulenaer BL, Bomans P \& Kockx MM 
(2005) Tuberculosis of the spine: CT and MR imaging features. JBR-BTR, 88 (2) 92-97

[11] Moore SL \& Rafii M (2001) Imaging of musculoskeletal and spinal tuberculosis. Radiol Clin North America, 39, 329-342. http://dx.doi.org/10.1016/S0033-8389(05)70280-3.

[12] Gautam VKS, Srivastava A, Khare A, Singh R (2014) CNS Tuberculoma mimicking brain metastasis. International journal of medicine, 2 (2), 68-70. http://dx.doi.org/10.14419/ijm.v2i2.3452.

[13] Trivedi R, Saksena S, Gupta RK (2009) Magnetic resonance imaging in central nervous system tuberculosis. The Indian Journal of Radiology \& Imaging.19 (4), 256-265. http://dx.doi.org/10.4103/0971-3026.57205.

[14] Gupta RK, Gupta S, Singh D, Sharma B, Kohli A \& Gujral RB (1994) MR imaging and angiography in tuberculous meningitis. Neuroradiology. 36:87-92. http://dx.doi.org/10.1007/BF00588066.

[15] Gupta RK, Kathuria MK \& Pradhan S (1999) Magnetization transfer MR imaging in CNS tuberculosis. AJNR Am J Neuroradiol.20867-75.

[16] Gupta R (2002) Magnetization transfer MR imaging in centra nervous system infections. Indian Journal Radiological Imaging. 1251-58.

[17] Bernaerts A, Vanhoenacker FM, Parizel PM, Van Goethem JW, Van Altena R, Laridon A, De Roeck J, Coeman V \& De Schepper AM (2003) Tuberculosis of the central nervous system: overview of neuroradiological findings. Eur. Radiol. 13:1876-1890. http://dx.doi.org/10.1007/s00330-002-1608-7.

[18] Jinkins JR, Gupta R,. Chang KH \& Rodriguez-Carbajal J (1995) MR imaging of central nervous system tuberculosis. Radiol. Clin. N. Am. 33:771-786.

[19] Kioumehr F, Dadsetan MR, Rooholamini SA \&. Au A (1994) Central nervous system tuberculosis: MRI. Neuroradiology 36:93-96. http://dx.doi.org/10.1007/BF00588067.

[20] Offenbacher H, Fazekas F, Schmidt R, Kleinert R, Payer F, Kleinert G \& Lechner H (1991) MRI in tuberculous meningoencephalitis: report of four cases and review of the neuroimaging lit$\begin{array}{llll}\text { erature. } & \text { J. } & \text { Neurol. } & \text { 238:340- }\end{array}$ http://dx.doi.org/10.1007/BF00315335.

[21] Kumar R, Kohli N, Thavnani H, Kumar A \& Sharma B (1996) Value of CT scan in the diagnosis of meningitis. Indian Pediatr. 33:465-468.

[22] Greenberg Mark S (2010) Handbook of Neurosurgery, $7^{\text {th }}$ Edition, Thieme

[23] Andronikou S \& Wieselthaler N (2004) Modern imaging of tuberculosis in children: thoracic, central nervous system and abdominal tuberculosis. Pediatr. $\quad$ Radiol. http://dx.doi.org/10.1007/s00247-004-1236-2.

[24] Przybojewski S, Andronikou S \& Wilmshurst J (2006) Objective CT criteria to determine the presence of abnormal basal enhancement in children with suspected tuberculous meningitis. Pediatr. Radiol. 36:687-696. http://dx.doi.org/10.1007/s00247-006-0160-z.

[25] Kamra P, Azad R, Prasad KN, Jha S, Pradhan S \& Gupta RK (2004) Infectious meningitis: prospective evaluation with magnetization transfer MRI. British Journal of Radiology, 77, 387-94. http://dx.doi.org/10.1259/bjr/23641059.

[26] Tandon PN, Bhatia R \& Bhargava S (1988) "Tuberculous meningitis" In: Vinken PJ, Bruyn GW, Klawans HZ, editors. Handbook of Clinical Neurology. Vol 8. Amsterdam: Elsevier; 196-226.

[27] Singh I, Haris M, Husain M, Husain N, Rastogi M \& Gupta RK ( 2008) Role of endoscopic third ventriculostomy in patients with communicating hydrocephalus: an evaluation by MR ventriculography. Neurosurgery Review, 31,319-325. http://dx.doi.org/10.1007/s10143-008-0137-5.

[28] Dastur DK, Lalitha VS, Udani PM \& Parekh U (1970) The brain and meninges in tuberculous meningitis-gross pathology in 100 cases and pathogenesis. Neurology India.18, 86-100.

[29] Shukla R, Abbas A, Kumar P, Gupta RK, Jha S \& Prasad KN (2008) Evaluation of cerebral infarction in tuberculous meningitis by diffusion weighted imaging. J Infect. 57, 298-306. http://dx.doi.org/10.1016/j.jinf.2008.07.012.

[30] Brismar J, Hugosson C, Larsson SG, Lundstedt C \& Nyman R (1996) Tuberculosis as a mimicker of brain tumor. Acta Radiol; 37:496-505. [PubMed: 8688230]

[31] Goyal M, Sharma A, Mishra NK, Gaikwad SB \& Sharma MC (1997). Imaging appearance of pachymeningeal tuberculosis. AJR Am J Roentgenol, 169:1421-4. [PubMed: 9353472] http://dx.doi.org/10.2214/ajr.169.5.9353472.

[32] Tandon PN \& Pathak SN (1973) Tuberculosis of the central nervous system. In: Spillane JD, editor. In Tropical Neurology. New York: Oxford University Press; 37-62.
[33] Gupta RK \& Lufkin RB (2001) MR imaging and spectroscopy of central nervous system infection; in Tuberculosis and other nontuberculous bacterial granulomatous infection. New York: Kluwer Academic, Plenum Publishers, 95-145 http://dx.doi.org/10.1007/b111688.

[34] Khoo JLS, Lau KY, Cheung CM \& Tsoi TH (2003) Central Nervous System Tuberculosis. J HK Coll Radiol, 6, 217-228

[35] Bargallo J, Berenguer J, Garcia-Barrionuevo J, Ubeda B, Bargallo N, Cardenal C \& Mercader JM (1996) The "target sign": is it a specific sign of CNS tuberculoma? Neuroradiology 38:547-550. http://dx.doi.org/10.1007/BF00626095.

[36] Poptani H, Gupta RK, Roy R, Pandey R, Jain VK \& Chhabra DK (1995) Characterization of intracranial mass lesions with in vivo proton MR spectroscopy. AJNR Am J Neuroradiol; 16:1593-603.

[37] Gupta RK, Jena A, Singh AK, Sharma A, Puri V \& Gupta M (1990) Role of magnetic resonance (MR) in the diagnosis and management of intracranial tuberculomas. Clin Radiol; 41:120-7. http://dx.doi.org/10.1016/S0009-9260(05)80143-6.

[38] Gupta RK, Pandey R, Khan EM, Mittal P, Gujral RB \& Chhabra DK (1993) intracranial tuberculomas: MRI signal intensity correlation with histopathology and localized proton spectroscopy. Magn Reson Imaging.; 11:443-9. $\quad$ http://dx.doi.org/10.1016/0730725X(93)90079-S.

[39] Gupta RK, Poptani H, Kohli A, Chhabra DK, Sharma B \& Gujral RB (1995) In vivo localized proton magnetic resonance spectroscopy of intracranial tuberculomas. Indian J Med Res; 101:19-24.

[40] Gupta RK, Roy R, Dev R, Husain M, Poptani H, Pandey R, Kishore J \& Bhaduri AP (1996) Finger printing of Mycobacterium tuberculosis in patients with intracranial tuberculomas by using in vivo, ex vivo, and in vitro magnetic resonance spectroscopy. Magn Reson Med; 36:829-33. http://dx.doi.org/10.1002/mrm.1910360605.

[41] Gupta RK \& Roy R (1999) MR imaging and spectroscopy of intracranial tuberculoma. Current Sci; 76:783-8.

[42] Whitener DR (1978) tuberculous brain abscess. Report of a case and review of the literature. Arch Neurol; 35, 148-85 http://dx.doi.org/10.1001/archneur.1978.00500270030007.

[43] Farrar DJ, Flanigan TP, Gordon NM, Gold RL, Rich JD (1997) Tuberculous brain abscess in a patient with HIV infection: case report and review. Am J Med; 102:297-301? [PubMed: 9217600] http://dx.doi.org/10.1016/S0002-9343(97)00386-0.

[44] Gupta RK, Vatsal DK, Husain N, Chawla S, Prasad KN, Roy R, Jha D \& Husain M (2001) Differentiation of tuberculous from pyogenic brain abscesses with in vivo proton MR spectroscopy and magnetization transfer MR imaging. AJNR Am J Neuroradiol.; 22:1503-9.

[45] Gautam VKS, Singh R \& Khurana S (2013) Brainstem Tuberculoma Presenting As Stroke. IOSR Journal of Dental and Medical Sciences (IOSR-JDMS). Volume 4, Issue 6, 18-19.

[46] Gautam VKS, Singh R \& Khurana S (2013) Transient Behavior Abnormality following injury to Splenium of corpus callosum during ventriculoperitoneal shunt Surgery. IOSR Journal of Dental and Medical Sciences (IOSR-JDMS).Volume 5, Issue 1, 26-29.

[47] VKS Gautam, Singh R \& Khurana S (2014) Hydrocephalus treated with VP shunt surgery: a clinical audit. International Journal of Health.2 (2), 26-29. http://dx.doi.org/10.14419/ijh.v2i2.2849.

[48] VKS Gautam, Singh R \& Khurana S (2014) Reviewing hydrocephalus: Personal experience of a Neurosurgeon with ventriculoperitoneal shunts treatment. International Journal of Health.2 ( 2) 2014, 56-59.

[49] Ersahin M, Hakan T, Ayan E, Berkman Z, Ekinci O, Ceran N \& Vardar Aker F (2010) Diagnostic and therapeutic role of CTguided stereotactic surgery in the managemnt of intracranial tuberculomas. Turkish neurosurgery, Vol: 20, No:3,295-302

[50] Report from the Meiacal Research Council Tuberculosis and Chest disease Unit (1980) National Suvey of tuberculosis notifications in Englnd and Wales 1978-79, Br. Med J; 281: 895-898.

[51] Gupta KB, Kumar A, Sen R, Sen J \&Verma M (2007) Role of ltrasonography and computed tomography in complicated cases of tuberculous cervical lymphadenitis. Indian Journal of Tuberculosis, 54;71-78.

[52] Gulati Y \& Gupta R (2005) Operative treatment of tuberculosis of dorsal and lumbar spine Apollo Medicine, June, Vol. 2, No. 2, 96100 .

[53] Kumar R (2005), Spinal tuberculosis: with reference to the children of northern India. Childs Nervous System. Jan, 21(1), 19-26. http://dx.doi.org/10.1007/s00381-004-1029-9.

[54] Murphy KJ, Brunberg JA, Quint DJ \& Kazanjian PH (1998) Spinal cord infection: myelitis and abscess formation. AJNR Am J Neuroradiol; 19:341-8. [PubMed: 9504492]

[55] Sharif HS, Aabed MY, Haddad MC (1992) "Magnetic resonance imaging and computed tomography of infectious spondylitis" In: 
Bloem JL, Satoris DJ, editors. MRI and CT of the musculoskeletal system: a text atlas. Baltimore: Williams and Wilkins; 1992. pp. 580-602.

[56] Brooks WD, Fletcher AP \& Wilson RR (1954) Spinal cord complications of tuberculous meningitis; a clinical and pathological study. Q J Med; 23:275-90. [PubMed: 13194846]

[57] Aiimsnets.org/ Spinalsurgery

[58] Jaiswal AK, Jaiswal S, Gupta SK, Singh Gautam VK \& Kumar S (2006) Intramedullary tuberculoma of the conus. J Clin Neurosci., Oct; 13(8):870-2. http://dx.doi.org/10.1016/j.jocn.2005.11.032. 